\title{
Identifying key elements of pediatric advance care planning with the help of bereaved parents and experienced care professionals
}

\author{
Kerstin Heina, Kathrin Knochela, Vedrana Zaimovica, Daniel Reimanna, Gian Domenico Borasiob, Monika Führera
}

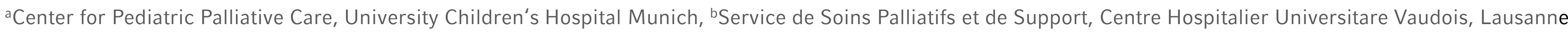

\section{Background}

Research has shown that advance care planning concepts for adults are not directly applicable to the pediatric setting. However, there are still few structured models for a specific pediatric advance care planning ( $\mathrm{PACP}$ ).

\section{Objective}

This study aimed at identifying key components of pACP taking into account the needs of all involved parties with the final goal of developing a pACP intervention for children with life-limiting diseases.

\section{Participants and Methods}

We selected bereaved parents, healthcare professionals (HCPs) and stakeholders of care networks by purposeful sampling and invited them to participate in a one-day transdisciplinary workshop.

During the workshop, participants were assigned to 1 of 3 focus groups according to their background (parents, facilitators, and implementators) to discuss their different perspectives and identify needs, barriers and helpful aspects of pACP.

Data were analyzed by content analysis.

\section{Results: Key elements of pediatric advance care planning}

\section{Timing}

Right time to start: As soon as possible, but considering parental readiness.

Iterative process: Discussions need to be repeated and documents updated in regular time intervals.

Sequential steps: Discussions should be embedded in continuous care of families considering emerging needs and increasing awareness and acceptance of the situation during disease trajectory.

\section{Decision-making discussions}

Parental decision-making:

- Ongoing communication process including discussions with palliative care team, partners and significant others.

- Parental deliberation is hardly visible for professionals. Thus, they often assume that parents are reluctant to make decisions.

Barriers:

- Insensitive communication

- Discussions at wrong times and places

- Unsuitable coping with emotions

- Professionals with lacking experience or knowledge about the child's disease.

Facilitating factors:

- Several meetings

- Time during and between meetings

- Multi-professional team to guide conversation

- Focus on the child

- Discuss hypothetical scenarios

- Open and non-judgmental discussions

\section{Documentation}

\section{Accompanying documentation}

- Minutes of discussions

- Journal for parents and patients (optional)

\section{Resulting documentation}

- Recommendations for procedures in case of emergencies

- Advance Directives
Supplementary information material

- Optional

- To be handed out conversation only after personal

\section{Implementation}

Barriers to provide care consistent with preferences:

- Disagreements between parents and professionals

- High emotional strain caused by forgoing resuscitation of children

- High emotional strain when missing social support during a crisis

- Concerns about parents suddenly changing their mind during a crisis

- Emergency physicians with little knowledge about the child, the underlying disease, and principles of palliative care

- Legal uncertainty of advance directives for children.

Facilitating factors:

- Personal conversation or round table to inform all care persons involved

- Designate responsible persons for emergencies

- Regular trainings

- Cooperation with pediatric palliative care team.

\section{Conclusion}

- We identified key components of pACP that take into account the needs of parents, HCPs and stakeholders of care networks.

- The key elements of pACP go beyond decision-making discussions and documents and also include their implementation.

- Identified elements of pACP served as a basis for the participative development of a specific pACP framework. 